\title{
Two-Dimensional Bragg-Ewald's Dynamical Diffraction and Spontaneous Gratings: Qualitative Nonlinear Optics Treatment
}

\author{
V.I. LYMAR* \\ V.N. Karazin Kharkov National University \\ 4, Svobody Square, 61077, Kharkov, Ukraine
}

\begin{abstract}
A stimulated light scattering in thin waveguide film, filled by point Kerr-type dipoles, under inclined pumping beam incidence is examined. The iterative procedure of successive approximations is applied to define qualitatively the space spectrum of waveguide mode scattered field at the condition of its self-consistency with dipole oscillations. A comparison with experimental data is discussed briefly.
\end{abstract}

PACS numbers: 42.25 .Fx

\section{Introduction}

Effects of stimulated, "light by light" scattering and four-wave mixing are the subjects of constant attention in nonlinear optics from the beginning [1, 2]. From practical viewpoint these processes are of particular interest in waveguides [3, 4]. Formation of so-called spontaneous gratings (SGs) on different plane surfaces and in waveguide films is studied to some extent independently as a specific process of the stimulated pumping beam scattering on scattered polariton or waveguide modes [5, 6]. Authors of [7] treated the SG formation as a result of phase conjugation process through degenerate four-wave mixing, but at normal pumping beam incidence only. We develop this approach for any incidence angle.

\section{Two-dimensional Ewald's problem}

Let us consider a thin waveguide (of one-mode thickness $t$ ) transparent film with permittivity $\varepsilon_{2}$, inside of which point identical Kerr-type dipoles are dis-

*e-mail: valentin.i.lymar@univer.kharkov.ua 


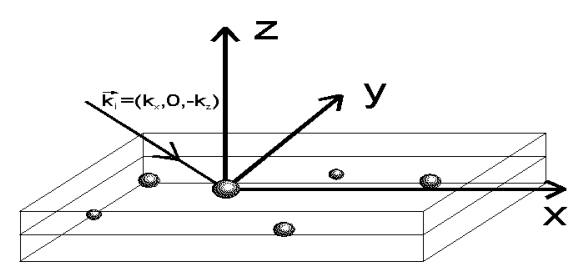

Fig. 1. The geometry of the problem. The wave vector of the reflected wave from the layer is not displayed for simplicity.

tributed with a density $n(\boldsymbol{R}), \boldsymbol{R}=(\boldsymbol{r}, z)=(x, y, z) . X Y$-plane is inside the layer at some depth $a(t=a+b)$. There is a pumping TM-wave with the wave vector $k_{i}=\left(k_{x},-k_{z}\right)$ in the $X Z$-plane of incidence (Fig. 1).

Permittivities of the upper and lower media are $\varepsilon_{1}$ and $\varepsilon_{3}$ accordingly. Under the influence of both incident and inevitable reflected waves dipoles transform into the sources of scattered radiation including waveguide modes. These waveguide modes influence in turn the dipole oscillations and so we receive a self-consistent problem similar to that which was studied by Ewald for a three-dimensional array of dipoles. Our problem can be reduced to two dimensions [8] by introducing the so-called "Sommerfeld's antenna oscillator force" $f(r)$ - a function integrating radiation of all point dipoles with the same $r$, but different $z$ (about cylindrical or Sommerfeld waves connected with SGs see also $[9,10]$ ). We shall treat the scattered waveguide TM-mode field only for the sake of simplicity. The $z$-component of the electric Hertzian vector potential for the scattered field inside the film $\Psi^{\mathrm{s}}(\boldsymbol{r}, z)$ has to satisfy the inhomogeneous Helmholtz equation

$$
\Delta \Psi^{\mathrm{s}}(\boldsymbol{r}, z)+\beta_{0}^{2} \Psi^{\mathrm{s}}(\boldsymbol{r}, z)=-4 \mathrm{i} Z(z) f(\boldsymbol{r}),
$$

here $\Delta=\frac{\partial^{2}}{\partial x^{2}}+\frac{\partial^{2}}{\partial y^{2}}-$ two-dimensional Laplace operator; $\beta_{0}-$ waveguide mode propagation constant; $Z(z)$ - known amplitude $z$-distribution for the waveguide TM-mode (see (4)). According to [8] "oscillator force" $f(r)$ is defined by the integral

$$
f(\boldsymbol{r})=\frac{2 \pi \beta_{0}}{\varepsilon_{2}^{2}(\mathrm{~d} \rho / \mathrm{d} \beta)_{\beta=\beta_{0}}} \int_{-b}^{a} n(\boldsymbol{r}, z) p_{z}(\boldsymbol{r}, z) Z(z) \mathrm{d} z,
$$

here $p_{z}(r, z)$ - the elementary dipole moment $z$-component; and functions $\rho(\beta)$ and $Z(z)$ have the forms

$$
\begin{aligned}
& \rho\left(\beta, t, \varepsilon_{1}, \varepsilon_{2}, \varepsilon_{3}\right)=\left(\frac{p}{\varepsilon_{3}}+\frac{q}{\varepsilon_{1}}\right) \cos (h t)+\mathrm{i}\left(\frac{h}{\varepsilon_{2}}+\frac{\varepsilon_{2} p q}{\varepsilon_{3} \varepsilon_{1} h}\right) \sin (h t) \\
& Z(z)=\cos \left[h_{0}(z+b)\right]+\mathrm{i} \frac{p_{0} \varepsilon_{2}}{h_{0} \varepsilon_{3}} \sin \left[h_{0}(z+b)\right] \\
& =\cos \left[h_{0}(a-z)\right]+\mathrm{i} \frac{q_{0} \varepsilon_{2}}{h_{0} \varepsilon_{1}} \sin \left[h_{0}(a-z)\right] \\
& \text { here } q=\sqrt{\varepsilon_{1} k_{0}^{2}-\beta^{2}}, h=\sqrt{\varepsilon_{2} k_{0}^{2}-\beta^{2}}, p=\sqrt{\varepsilon_{3} k_{0}^{2}-\beta^{2}} \text { in } \\
& p_{0}=-\mathrm{i} \sqrt{\beta_{0}^{2}-\varepsilon_{3} k_{0}^{2}}, q_{0}=-\mathrm{i} \sqrt{\beta_{0}^{2}-\varepsilon_{1} k_{0}^{2}} \text { in }(4), k_{0}=\omega / c \text {. and }
\end{aligned}
$$


In the first linear approach $p_{z}^{(1)}=\alpha_{z m} E_{m}^{\text {ext }}, \alpha_{l m}$ - the elementary dipole linear polarizability tensor; $E_{m}^{\text {ext }}=E_{m}^{\text {inc }}+E_{m}^{\text {ref }}$ - the external electric field components, connected in the known way with the vector potential $z$-component $\Psi^{\text {ext }}(r, z)=\mathrm{e}^{-\mathrm{i} \boldsymbol{k}_{x} \boldsymbol{r}}\left(I \mathrm{e}^{\mathrm{i} k_{z} z}+R \mathrm{e}^{-\mathrm{i} k_{z} z}\right)$ for incident and reflected TM-polarized waves $I$ and $R$ - their corresponding complex amplitudes, which can be considered as known for our one-layered configuration. We have in the first approach [8]:

$$
\Psi^{\mathrm{s} 1}(\boldsymbol{r}, z)=4 \mathrm{i} Z(z) \quad \int_{\left(\beta_{\mathrm{s} x}, \beta_{\mathrm{s} y}\right)} \frac{F^{(1)}\left(\boldsymbol{R}_{\mathrm{s}}\right)}{\left[\boldsymbol{R}_{\mathrm{s}}^{2}-\beta_{0}^{2}\right]} \mathrm{e}^{\mathrm{i} \boldsymbol{R}_{\mathrm{s}} \boldsymbol{r}} \mathrm{d} \boldsymbol{R}_{\mathrm{s}},
$$

where $F^{(1)}\left(\boldsymbol{R}_{\mathrm{s}}\right)=\frac{1}{(2 \pi)^{2}} \int_{(x, y)} f^{(1)}(\boldsymbol{r}) \mathrm{e}^{\mathrm{i} R_{\mathrm{s}} \boldsymbol{r} \mathrm{d} r}$ - linear "structure scattering factor"; $\beta_{\mathrm{s}}$ - scattered wave vector component in $X Y$-plane; $f^{(1)}(\boldsymbol{r})$ is found using $p_{z}^{(1)}(r, z)$ and (2). Equation (5) can be rewritten in the form of curve-line integral along a circle of radius $\beta_{0}$ in the wave vector $\left(\beta_{x}, \beta_{y}\right)$-plane (if $F^{(1)}\left(\beta_{\mathrm{s}}\right)$ is a sufficiently smooth function and $\beta_{0} \gg g, g$ is an imaginary part of $\beta_{0}$ ):

$$
\Psi^{\mathrm{s} 1}(\boldsymbol{r}, z)=2 \pi Z(z) \oint_{\beta_{0}} F^{(1)}\left(\boldsymbol{R}_{0}\right) \mathrm{e}^{-\mathrm{i} \boldsymbol{R}_{0} \boldsymbol{r}} \mathrm{d} \beta,
$$

here $\beta_{0}$ - wave vector of the scattered waveguide mode.

The second approach $\Psi^{\mathrm{s} 2}(\boldsymbol{r}, z)$ can be received taking into account nonlinear polarization waves because of Kerr-type nonlinearity of every point dipole

$$
\begin{aligned}
& p_{z}^{(2)}=\sigma_{z l m n}(\omega)\left[2 E_{1}^{\mathrm{ext}} E_{m}^{\mathrm{ext} *} E_{n}^{\mathrm{s} 1}+\left(E_{1}^{\mathrm{inc}} E_{m}^{\mathrm{ext}}+E_{1}^{\mathrm{ext}} E_{m}^{\mathrm{ref}}\right) E_{n}^{\mathrm{s} 1 *}\right], \\
& \sigma_{z l m n}=\chi_{z l m n}(\omega ;-\omega, \omega, \omega)+\chi_{z l m n}(\omega ; \omega,-\omega, \omega)+\chi_{z l m n}(\omega ; \omega, \omega,-\omega),
\end{aligned}
$$

here $\chi_{z l m n}$ - cubic nonlinear dipole polarizability tensor; $E_{n}^{\text {s1 }}$ - electric field components of scattered waveguide modes, calculated from the $\Psi^{s 1}(r, z)(5),(6)$. For the dynamical grating, Eq. (7) corresponds to self-diffraction process of $\boldsymbol{k}_{x}$-wave by $\left(\boldsymbol{\beta}_{0}-\boldsymbol{k}_{x}\right)$-grating both into positive order (first term) forming waveguide mode obligingly and into negative order forming radiative, nonradiative modes and, that is most important, pair of conjugated wa veguide modes (see lower). All Eqs. (1)-(6) are valid for the second approach $\Psi^{\mathrm{s} 2}(r, z)$ also after replacing of the upper index "1" by "2". Substituting $p_{z}^{(2)}$ into (2) we can calculate $F^{(2)}\left(\boldsymbol{\beta}_{\mathrm{s}}\right)$ and get the phase synchronization conditions

$$
\boldsymbol{\beta}_{\mathrm{s}}=\boldsymbol{\beta}_{0} \quad \text { and } \quad \boldsymbol{\beta}_{\mathrm{s}}=2 \boldsymbol{k}_{x}-\boldsymbol{\beta}_{0} .
$$

These conditions state two circles with centers at $O$ and $2 k_{x}$ in the reciprocal $\left(\beta_{x}, \beta_{y}\right)$-wave vector space (see the polar diagram sketched in Fig. 2). Using (5), (6) we receive the result for $\Psi^{\mathrm{s} 2}(\boldsymbol{r}, z)$ together with the additional condition $\beta_{\mathrm{s}}=\beta_{0}$. In physics words the second approach predicts an amplification of both all scattered waveguide modes and especially two conjugated waveguide modes, corresponding to points $C$ and $C^{\prime}$ (Fig. 2). The latter corresponds to the four-wave mixing process, shown by the triangle $O C 2 k_{x}$ :

$$
\boldsymbol{\beta}_{C}+\boldsymbol{\beta}_{C^{\prime}}=k_{x}+k_{x} .
$$


In media with almost local response it is the conjugation process that will be overwhelming $[1,2]$, and therefore a considerable gain in amplitude of waveguide modes with wave vectors $\beta_{C}$ and $\beta_{C^{\prime}}$ will be obtained for that case.

For the third approach $\Psi^{\mathrm{s} 3}(\boldsymbol{r}, z)$ we must take into account $\boldsymbol{\beta}_{C}$ and $\boldsymbol{\beta}_{C^{\prime}}$ waveguide modes also along with pumping $\boldsymbol{k}_{x}$-wave (however in what follows, we assume the pumping wave amplitude far beyond that of scattered modes). Expression for $p_{z}^{(3)}(\boldsymbol{r}, z)$ is bound to contain the terms representing products of the external field by the field of waveguide mode $\boldsymbol{\beta}_{C}$ or $\boldsymbol{\beta}_{C}$ and by the scattered waveguide mode field obtained at the first approximation. The phase synchronization conditions for scattered waves connected with those terms

$$
\boldsymbol{\beta}_{\mathrm{s}}=\boldsymbol{k}_{x} 6 \boldsymbol{\beta}_{C} 7 \boldsymbol{\beta}_{0} \text { and } \boldsymbol{\beta}_{\mathrm{s}}=\boldsymbol{k}_{x} 6 \boldsymbol{\beta}_{C^{\prime}} 7 \boldsymbol{\beta}_{0}
$$

produce four further circles in the reciprocal $\left(\beta_{x}, \beta_{y}\right)$-wave vector space (Fig. 2 ). The points of intersection of these circles and two previous ones correspond to four-wave mixing processes with the waveguide modes conjugation, for example,

$$
\boldsymbol{\beta}_{S 1}+\boldsymbol{\beta}_{S 1^{\prime}}=\boldsymbol{k}_{x}+\boldsymbol{\beta}_{C}, \quad \boldsymbol{\beta}_{S 2}+\boldsymbol{\beta}_{S 2^{\prime}}=\boldsymbol{k}_{x}-\boldsymbol{\beta}_{C^{\prime}}
$$

and so on. A substantial gain is possible for these waveguide modes and analogous to them. As an example, in Fig. 2 there is shown the quadrangle $O S_{1} A C$ relevant to the first of Eqs. (11).

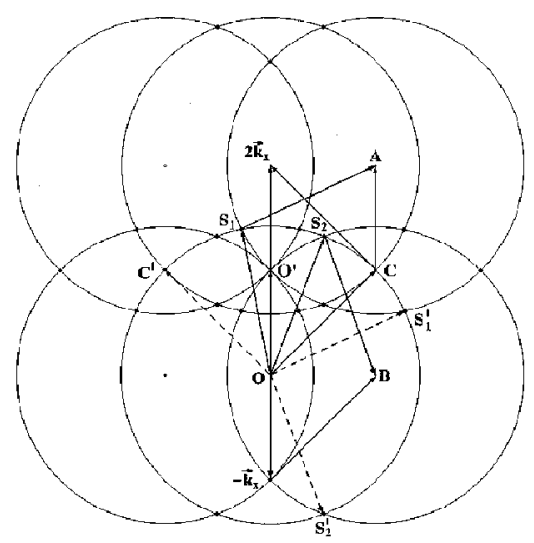

Fig. 2. The polar diagram for wave vectors of the stimulated scattered waves within second and third approach. $X$-axis tends vertically.

Finally, according to general theory of four-wave mixing [11], an amplification of some mode at the process could be treated as a result of a pumping wave diffraction on the dynamical grating formed by another pumping wave and conjugated mode, which is amplifying also. Then, for our case, the four-wave mixing process development is equivalent to the appearance of SGs with grating vectors counted from the point $O^{\prime}$ in the reciprocal wave vector space (Fig. 2), i.e. the following equation is valid: 


$$
\boldsymbol{K}=\boldsymbol{\beta}_{\mathrm{s}}-\boldsymbol{k}_{x}
$$

where $\boldsymbol{\beta}_{\mathrm{s}}$ is a wave vector component in $X Y$-plane of the amplified scattered wave, $\boldsymbol{K}$ - corresponding grating vector. Thus the polar diagram in Fig. 2 presents not only the spectrum of scattered waves, but the gratings ones also.

\section{Comparison with experimental data}

At the experiment under consideration SGs were recorded in AgCl-waveguide film ( $t \approx 120 \mathrm{~nm}$ ), doped by fine Ag-granules, under irradiation by two-beam pumping $(\lambda=633 \mathrm{~nm})$ at $60^{\circ}$-angle of incidence. After fixing the SGs diffraction spectrum could be obtained at normal incidence of the reconstructing laser beam (for more details, see [12]). The photography in Fig. 3 shows such spectrum on the plane screen.

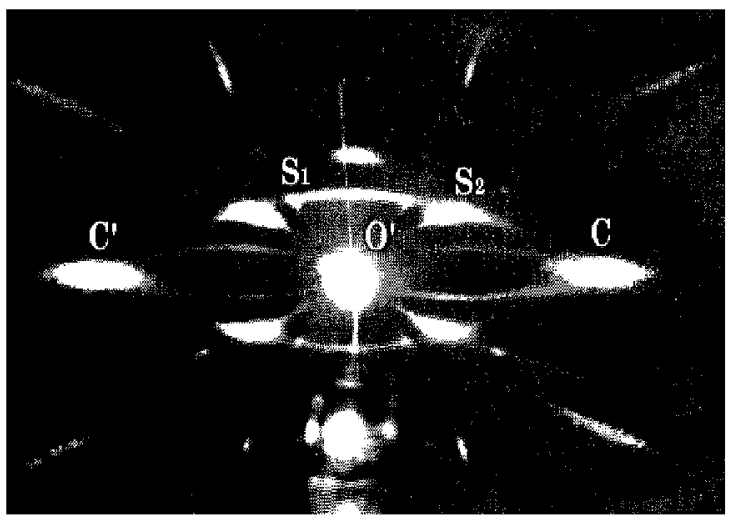

Fig. 3. The diffraction picture from SGs at normal incidence of reconstructing laser beam. SGs were recorded previously on $\mathrm{TM}_{0}$-modes under oblique incidence of the pumping laser beam on photosensitive waveguide film. The literal symbols and axis directions are consistent with that of Fig. 2.

There is a general qualitative agreement between experimental spectrum and that has been obtained from the theoretical model. Points similar to $S_{1}^{\prime}$ or $S_{2}^{\prime}$ can be observed, but only at inclined incidence of the reconstructing beam because of small periods of corresponding gratings.

To explain a significant difference in the progress of $S_{2}$-grating with respect to $S_{1}$-grating, the availability of pumping $\boldsymbol{k}_{x}$-wave during two-beam pumping conditions has to be taken into account. In addition to the conjugation process in terms of the second of Eqs. (11), the following process could occur:

$$
\boldsymbol{\beta}_{S 2}+\boldsymbol{\beta}_{S 2^{\prime}}=\boldsymbol{\beta}_{C}-\boldsymbol{k}_{x},
$$

shown by the quadrangle $O S_{2} B\left(-\boldsymbol{k}_{x}\right)$ in Fig. 2. Similar process for modes of type $\boldsymbol{\beta}_{S 1}$ and $\boldsymbol{\beta}_{S 1^{\prime}}$ is impossible. Besides, the modes $\boldsymbol{\beta}_{S 2}$ and $\boldsymbol{\beta}_{S 2^{\prime}}$ are, in some sense, 
"more collinear" than the modes $\boldsymbol{\beta}_{S 1}$ and $\boldsymbol{\beta}_{S 1^{\prime}}$. But it is known that a conjugation efficiency is stronger in the collinear four-wave-mixing geometry.

Sufficiently distinct reflexes near upper and lower edges of the photography in Fig. 3 could be explained if, for the third approach, we would not suppose that the amplitudes of $\boldsymbol{\beta}_{C}$ and $\boldsymbol{\beta}_{C^{\prime}}$ waveguide modes are less important than the amplitude of pumping $\boldsymbol{k}_{x^{-}}$-wave. Then circles at centres $\boldsymbol{\beta}_{C} 6 \boldsymbol{\beta}_{C^{\prime}}$ and $\boldsymbol{\beta}_{C^{\prime}} 6 \boldsymbol{\beta}_{C}$ would appear in the polar diagram in Fig. 2 and additional four-wave mixing processes with the waveguide mode conjugation would be included into theoretical treatment.

The distinct reflex slightly above the arc $S_{1} S_{2}$ (see diffraction pattern of Fig. 3) together with peripheral degraded reflexes, as well as arcs $C^{\prime} O^{\prime}$ and $O^{\prime} C$, yield description within the fourth approximation. Analogously to former analysis we must take into account fields of waveguide modes similar to $\boldsymbol{\beta}_{S 2}$ on the level with the field of pumping $\boldsymbol{k}_{x}$-wave, as well as the fields of $\boldsymbol{\beta}_{C}$ and $\boldsymbol{\beta}_{C^{\prime}}$ waveguide modes. But for the time being we will not discuss this question in more detail.

It is matter to note that the amplification of arcs, i.e. of waveguide modes outside the conjugation process, is impossible in media with the local response under the one-beam pumping. This limitation is taken off in a medium with the nonlocal response or during the two-beam pumping, when four-wave mixing process is possible for any waveguide modes opposing one another. The observability of the arcs in our experiment is caused by rather both circumstances.

At the first view, there are some essential differences between the layer with Kerr-type dipoles, examined as the theoretical model, and the photosensitive film, applied in the experiment. Firstly, the dynamical gratings of the model are completely reversible, while similar to another SGs [5, 9], gratings in $\mathrm{AgCl}-\mathrm{Ag}$ films are irreversible. But to our mind, this distinction is not principal. At our conditions the SGs irreversibility could be eliminated under laser recording with a simultaneous using of an auxiliary wipeouting noncoherent irradiation. Secondly, the growth of real gratings is much slower than instantaneous response of Kerr-type dipoles. This distinction is due to the mechanism of the nonlinear response of the $\mathrm{AgCl}-\mathrm{Ag}$ film. Quantitative results can be dependent on that circumstance rather than qualitative ones. The quantitative experimental estimation of the effective nonlinear susceptibility of the $\mathrm{AgCl}-\mathrm{Ag}$ films is a subject of subsequent investigations.

\section{Conclusions}

The developed above theoretical treatment demonstrates that the phenomena of the origin and of the growth of the SGs, formed in waveguide films or on surfaces of solids as a result of stimulated scattering of inducing laser beam into waveguide or polariton modes, can be described within degenerate four-wave-mixing interaction at any laser beam incidence angle. All of the elements of the SGs diffraction pattern are explained qualitatively. 


\section{Acknowledgments}

Author is thankful very much to Prof. V.K. Miloslavsky and to Prof. L.A. Ageev, as well as Yu.V. Lisenko and F.S. Novokhatsky for fruitful discussions.

Author appreciates sincerely all organizers of the nonlinear optics conferences in Świnoujście-2000 and Łukęcin-2002, especially Prof. E. WeinertRączka and Prof. A.D. Boardman, for invitations and hospitality at these meetings. They have extended substantially author's horizons.

Author would like to devote this work for the light memory about wonderful Ukrainian physics teacher A.O. Fedorchenko, man of high mind and great heart. The world is another without him, but his love to people and to physics is inspiring for us.

\section{References}

[1] R.Y. Chiao, P.L. Kelley, E. Garmire, Phys. Rev. Lett. 17, 1158 (1996).

[2] S. Odoulov, K. Belabaev, I. Kiseleva, Opt. Lett. 10, 31 (1985).

[3] E. Weinert-Raczka, Opt. Commun. 49, 245 (1984).

[4] G.I. Stegeman, C.T. Seaton, C. Karaguleff, IEEE J. Quantum Electron. QE-22, 1344 (1986).

[5] A.E. Siegman, P.M. Fauchet, IEEE J. Quantum Electron. QE-22, 1384 (1986).

[6] L.A. Ageev, V.K. Miloslavsky, Opt. Eng. 34, 960 (1995).

[7] B.D. Robert, J.E. Sipe, J. Opt. Soc. Am. B 8, 786 (1991).

[8] V.I. Lymar, in: Soliton-Driven Photonics, Eds. A.D. Boardman, A.P. Sukhorukov, Vol. 31, Kluwer, Dordrecht 2001, p. 363.

[9] V.A. Kosobukin, Solid State Phys. 35, 884 (1993) (in Russian).

[10] V.A. Kosobukin, M.N. Libenson, A.G. Rumyantsev, Opt. Spektrosk. 65, 948 (1988)

[11] Y.R. Shen, The Principles of Nonlinear Optics, Wiley, New York 1984.

[12] L.A. Ageev, V.I. Lymar, V.K. Miloslavsky, Opt. Spektrosk. 69, 1119 (1990). 